\title{
L'anthropologie, la recherche et l'intervention sur le sida : enjeux méthodologiques d'une rencontre
}

\section{Laurent Vidal}

\section{OpenEdition}

\section{Journals}

Édition électronique

URL : http://journals.openedition.org/apad/1982

DOI : 10.4000/apad.1982

ISSN : 1950-6929

Éditeur

LIT Verlag

Édition imprimée

Date de publication : 1 décembre 1994

Référence électronique

Laurent Vidal, « L'anthropologie, la recherche et l'intervention sur le sida : enjeux méthodologiques d'une rencontre », Bulletin de l'APAD [En ligne], 8 | 1994, mis en ligne le 23 novembre 2007, consulté le 07 septembre 2020. URL : http://journals.openedition.org/apad/1982 ; DOI : https://doi.org/10.4000/ apad.1982

Ce document a été généré automatiquement le 7 septembre 2020.

Bulletin de l'APAD 


\title{
L'anthropologie, la recherche et l'intervention sur le sida : enjeux méthodologiques d'une rencontre
}

\author{
Laurent Vidal
}

Politique, anthropologie et Sida : introduction à une rencontreRepères de l'anthropologie en politique

1 L'histoire de l'anthropologie offre de multiples exemples de sollicitations de son regard et de ses analyses émanant de disciplines des "sciences exactes" mais, aussi et surtout, de pouvoirs et institutions politiques ou économiques. Depuis quelques années, la revue "Gradhiva" nous propose des témoignages variés de ce lien, prenant la forme d'une demande, entre le pouvoir politique et la recherche anthropologique. Du sociologue P. Descamps qui, en 1930, répond à la commande de Salazar, alors Ministre des finances du Portugal, de "livrer un diagnostic sur l'état social du Portugal" (Kalaora, 1989, p.52) aux travaux de Loria, en Italie, utilisés par les militaires italiens en Erythrée dans le contexte d'une anthropologie "consentante et même intégrée dans l'idéologie autoritaire et raciste du fascisme" (Puccini, 1991, p. 64-5), se dessine l'institutionnalisation de l'anthropologie au contact étroit du pouvoir politique.

2 Ces rencontres, au-delà du résultat d'affinités idéologiques, ont constitué des lieux d'expérimentation de l'analyse anthropologique et de sa pratique méthodologique. Ainsi, alors que S. Puccini remarque que les "Istruzioni" de Loria "correspondent à ce qui sera plus tard connu sous le terme "applied anthropology'" (p.75), les "rapports ethnographiques" de Jorge Diaz sur les colonies portugaises en Afrique demeurent, sous la forme de compte-rendus au Ministre d'Outre-mer, de véritables "œuvres scientifiques" (De Pina Cabral, 1992, p.40). L'anthropologie française a en grande partie elle aussi fondé son développement sur des réponses aux interrogations du pouvoir central: des "monographies" de commande aux "rapports de tournée" des administrateurs coloniaux, un corpus de connaissances s'est constitué, encore largement exploité et valorisé par les anthropologues et historiens africanistes. 
3 Actuellement, la "demande d'ethnologie" évoquée par G. Althabé et provenant de l'entreprise, de la ville (pour les opérations d'urbanisme, en particulier) mais aussi, pourrait-on ajouter, de la santé, serait "le signe des transformations internes aux Sciences sociales" marquées par "l'effacement des perspectives explicatives globales et (...) la désignation de pratiques du quotidien comme domaine principal de la recherche" (Althabe, 1993, pp.89-90). Ce retour au "terrain" - qui ne nous semble pas avoir été délaissé par les anthropologues eux-mêmes mais qui, plutôt, retrouve une pertinence scientifique aux yeux des sollicitations extérieures en appelant à cette démarche - revêt une dimension particulière lorsqu'il s'applique à des problèmes de santé publique. A cet égard, le détour introductif par quelques exemples de collaboration - fructueuse pour les deux parties - de l'anthropologie avec le monde politique trouve, dans des proportions et pour des enjeux apparemment peu comparables, sa justification dans le cadre de notre propos sur la rencontre entre perspectives biomédicales et anthropologiques autour du problème du Sida.

Dilemmes de l'anthropologie face au Sida

4 Les fondements et les implications politiques du Sida ne sont plus à démontrer : des anthropologues (Dozon et Fassin, 1988 ; Seidel, 1993) et des médecins (au premier rang desquels J. Mann, ancien responsable du Grand programme Sida de l'OMS ; Mann, 1993) se rejoignent pour désigner les obstacles politiques (reconnaissance de l'épidémie par les Etats, disponibilité de moyens financiers, respect des droits de l'homme) à une prévention efficace de la maladie. La concordance des diagnostics de la médecine et des Sciences sociales sur les lacunes de l'intervention des responsables politiques dans la lutte contre le Sida se trouve singulièrement atténuée par la difficile mise en pratique des activités de recherche pluridisciplinaires susceptibles de répondre aux questions posées par le développement de l'épidémie. Sur ce point essentiel de la réflexion sur le Sida, étendu à l'ensemble des problèmes sanitaires rapprochant anthropologues et médecins, J.L. Jarnard pose le constat de l'existence d"'urgences indiscutables qui, plus d'une fois néanmoins, et c'est fâcheux, induisent à reléguer au second plan, voire sous le boisseau - délibérément ou non - les complexes causalités imputables aux systèmes de santé vernaculaires susceptibles d'expliquer "quelques faillites de ces recherches appliquées, et admet que "c'est dans la pluridisciplinarité et sa difficile distribution des rôles que s'établissent les "anthropologies scientifiques" (Jarnard, 1989-90, pp.46-47). $C^{\prime}$ 'est de cette nécessaire et parfois impossible pluridisciplinarité - dans le sens où les obstacles épistémologiques, méthodologiques et éthiques en découragent la réalisation - que nous souhaiterions débattre, à partir du cas exemplaire de l'approche du Sida.

5 Avant de les présenter plus en détail, il convient dans un premier temps de se pencher sur les principaux éléments du problème de la collaboration entre médecine et anthropologie. Une première interrogation s'impose : la sollicitation par les Sciences biomédicales n'est-elle pas fondée - pour reprendre un terme d'épidémiologie - sur un "biais", fruit d'un malentendu ? En effet, face à l"'urgence" - qui a maintenant plus d'une décennie... - constituée par la progression du Sida, la médecine recherche des analyses, des appréciations sur des comportements (en général "sexuels"), des situations sociales, sans pour autant assumer et accepter les méthodes en mesure de les produire dans une perspective anthropologique. Des compromis ont été trouvés (enquêtes CACP, "Connaissances, attitudes, croyances et pratiques", KAPB en anglais ; RP, "Relations entre partenaires"; et, moins systématiquement, RAP, "Rapid assessments procedures") mais qui, malgré leurs mises à contribution de sociologues ou 
d'anthropologues, ne paraissent pas satisfaisantes au regard d'une conception classique de l'anthropologie. Dès lors, la rencontre entre médecins (et acteurs de la santé en général : chercheurs et praticiens confondus) et anthropologues ne peut-elle s'effectuer que "par défaut" absence de représentativité des informations recueillies par les anthropologues - d'où leur impossible application -, ou abandon de l'approche anthropologique proprement dite ? Répondre à un tel dilemme revient, en partie, à se demander si les exigences - intransigeances ? - méthodologiques de l'anthropologie ne sont pas obsolètes dans le cas d'un problème de santé publique qui mobilise l'ensemble de la communauté scientifique. Il s'agit là d'un problème épistémologique, plus généralement lié à l'évolution de la pensée anthropologique relative au rôle de cette science sociale dans l'appréciation de l'intervention face aux enjeux sociaux de notre époque, comme le Sida.

Une fois cernés les enjeux épistémologiques de cette remise en cause, on peut toutefois se demander si une discussion puis un abandon des méthodes qui ont donné un corpus de connaissances, base du savoir anthropologique, ne risque pas de remettre en question les présupposés éthiques et conceptuels de la discipline. En d'autres termes, peut-on rejeter des principes méthodologiques sans, ipso facto, contredire des choix conceptuels et éthiques ? Nous verrons ainsi que sur la question de l'éthique de la recherche en anthropologie, à travers sa confrontation avec la problématique du Sida et sa perception par la biomédecine, peut s'avérer enrichissante. En revanche, la pratique d'une pluridisciplinarité tendant à uniformiser les options méthodologiques au détriment de la démarche anthropologique de l'observation et du dialogue dans la durée, s'accompagne d'un appauvrissement de la conceptualisation des objets de l'anthropologie au cœur de la réflexion sur le Sida (la maladie, la guérison, la sexualité, l'altérité).

Questions de méthode : regard anthropologique sur des choix d'enquêteLimites anthropologiques des enquêtes "socio-comportementales".

7 Lorsque le Grand Programme Sida de l'OMS publia, entre novembre 1989 et février 1990, ses premiers questionnaires et guides d'entretien pour les enquêtes CACP et "Relations entre partenaires" (RP) (OMS, 1989 - a, b, c, d et 1990- a, b), l'objectif est donc d'appréhender les représentations et comportements (sexuels) face au Sida. En eux-mêmes, ces thèmes de réflexion concernent directement l'anthropologie, sollicitée - dès lors que la transmission hétérosexuelle du VIH s'est confirmée en Afrique (Piot, 1984) - pour fournir des informations sur les comportements sexuels dits "à risque" des populations côtoyées depuis des décennies. D'autre part, le concept de représentation (de la maladie, du malheur, de la mort) est au centre de préoccupations d'une anthropologie formalisée notamment par M. Augé (1984, 1986), J.P. Dozon (1983), A. Zempleni (1982) et A. Sindzingre (et A. Zempleni, 1982). Cet ancrage de la perspective de l'OMS dans celle de l'anthropologie ne doit cependant pas masquer de profondes divergences dans l'approche des questions de représentations et de comportements qui rendent délicate la contribution de l'anthropologue.

8 A cet égard, M. Carael, sociologue à l'OMS, justifie la difficulté de la rencontre et de la collaboration avec l'anthropologie par les objectifs que s'était assignée l'OMS lors du lancement des enquêtes CACP. Le choix d'enquêtes quantitatives traduisait la volonté d'une visée descriptive (des connaissances sur le Sida, des comportements "à risque") en l'absence de "cadre théorique dans lequel fonctionner" et compte-tenu de l'"urgence de la situation" (Carael, 1993, p. 28). D'emblée, l'apport de l'anthropologie 
ne semble pas recherché : la littérature sur des thèmes utiles à l'analyse de la sexualité (le mariage, par exemple) n'est pas prise en considération ${ }^{1}$ et le concept $d$ "'urgence" sied mal à la perspective anthropologique inscrite dans la durée. Or, si l'on excepte l'objectif de "mesurer des fréquences de comportements" - peu susceptible de mobiliser un anthropologue - la volonté d' "évaluer des interventions, des activités, des connaissances" dans une optique comparative ("multisite" et "multiculturelle") s'accorde avec une problématique anthropologique actuelle, développée notamment dans les études sur les systèmes de santé (Hours, 1993). De même, l'application d'enquêtes CACP à des groupes spécifiques (adolescents, personnels de santé, prostituées et clients) peut s'adapter avec le regard ciblé sur une communauté donnée prôné par l'anthropologue.

9 Parallèlement à ces points de convergence entre les objectifs des enquêtes CACP et les préoccupations de l'anthropologie, l'OMS soutient des options discutables sur le plan de l'appréhension de l'objet de recherche (les phénomènes de connaissance et de représentations).

M. Carael mentionne en effet le souhait, à l'OMS, "résister à la tentation d'explorer les motivations parce que les modèles rationalistes de comportements humains partent d'une série d'hypothèses que nous, à l'OMS, ne partageons pas : (i.e.) que les répondants sont conscients de leurs motivations personnelles, (...) ont la volonté de déclarer leurs raisons, (...) ont un choix" (Carael, 1993, p.31). La question du "pourquoi ?" n'est donc jamais posée. L'argument de la non-conscience que l'individu aurait de son attitude me parait découler, en réalité, du type de contact établi avec l"'enquêté" : fondé sur un unique entretien, superficiel, il ne permet pas, par définition, d'approcher les motivations, perceptibles à travers la seule répétition des échanges, la mise en confiance (qui a certes, elle aussi, ses limites) des interlocuteurs.

Les questionnaires "CACP" et "RP"

Plus fondamentalement, se pose ici le problème de la validité du "déclaratif sur des thèmes aussi précis et personnels que la vie sexuelle. Là encore, l'approche quantitative se heurte à une difficulté inhérente au "protocole" de l'étude. Les manuels de présentations des études CACP et le questionnaire standard de l'OMS témoignent des insuffisances, sur le plan anthropologique, de cette approche des attitudes se rapportant au Sida. Dans le questionnaire servant de canevas aux enquêtes engagées en Afrique (OMS, 1989 - d), des questions inutiles ou insuffisamment précises nuisent à la pertinence du projet. Ainsi, la simple interrogation "quelle est votre religion ?" (Q 113, p.6) ne présente guère d'intérêt sous cette forme. Nous savons les systèmes de croyances et de pratiques religieuses suffisamment complexes et "cumulables" pour rejeter une approche aussi vague du sentiment religieux. A vouloir explorer cette dimension religieuse autant y procéder méthodiquement, en évitant des questions qui, pour être habituelles, n'en sont pas moins dénuées de sens. Si l'on souhaite établir de véritables corrélations avec les comportements face au Sida, il conviendrait, dans un premier temps, d'analyser la perception de la religion (critères d'affiliation, nature de la croyance et de la pratique) qu'en ont les personnes interrogées.

Toujours dans la section sur les "caractéristiques individuelles", la question "Est-ce que votre conjoint/partenaire régulier a d'autres partenaires réguliers ? Si oui, combien ?" ( $Q$ 121, p.6) suppose de façon étonnante et quelque peu naïve que la vie extra-conjugale du partenaire est connue de la personne interrogée. L'information recherchée (le nombre de partenaires sexuels) n'est pas ici en cause mais bien la démarche adoptée, 
vouée à l'échec. Dans le même ordre d'idées, l'existence de deux questions ouvertes (1. "Quelle est d'après vous la plus grave maladie, problème de santé auquel est confronté le monde (ou, 2"votre pays"... aujourd'hui ?") dans le chapitre "conscience du Sida" (Q201 et 202, P.9) ne se justifie nullement Qu'apporte en effet l'identification du "principal problème de santé du monde" selon l'enquêté ? Que peut-on répondre à ce type de question: le cancer, le Sida, les maladies cardio-vasculaires? Une question fermée aurait été, ici, plus pertinente :

13 L'approche spécifique des connaissances et attitudes sur le Sida comprend aussi des questions superflues ou déplacées. Ainsi, imagine-t-on une réponse négative à la question "pensez-vous qu'une personne puisse attraper le Sida avec quelqu'un qui a cette maladie ?" (Q 209, p.9) ou, encore, l'affirmation d'un risque élevé pour soi-même d'attraper le Sida?

Répondre "des 'chances' importantes" à la question fermée "quelles sont vos 'chances' d'attraper le Sida ? diriez-vous que ce sont : des chances..." reviendrait à assumer un comportement "à risque" (objet en lui-même d'une série indépendante de questions) et le refus ou l'impossibilité de le modifier. Il s'agit là d'attitudes psychologiques certes possibles mais qui paraissent difficilement identifiables par une approche aussi directe, alors qu'un recoupement de réflexions sur le comportement sexuel serait en mesure de fournir ces indications sur la conscience individuelle du risque.

La question ouverte "Qu'est-ce qui cause le Sida ?" (Q 207, p.9) interdit l'identification des différents niveaux de causalité en œuvre dans la perception de l'origine de la maladie (Vidal, 1992 - a, b). L'enquêteur obtiendra une énumération de causes alors qu'il serait bien plus instructif d'en connaître la succession et l'importance respective (agression en sorcellerie puis relation sexuelle "à risque", par exemple). Sur le thème général de l'origine du mal ou du malheur, il est demandé d'apprécier une série d'affirmations parmi lesquelles "si quelque chose de mal m'arrive dans la vie, je fais comme si ce n'était pas de ma faute mais juste que c'est ainsi que les choses sont" (réponses possibles: "d'accord", "pas d'accord"; Q 809, p.24). Si la personne marque son accord avec cette réflexion le questionnaire n'offre malheureusement pas la possibilité d'explorer le problème de la "fatalité", notamment dans les implications qu'elle sous-tend (caractère divin de la sanction ou mise en cause, au-delà d'un discours fataliste, de personnes clairement identifiées).

16 En se penchant sur ces quelques exemples de questions soit inutiles, soit imprécises, on conçoit que toute collaboration des anthropologues et plus simplement toute exploitation des données recueillies suivant ces protocoles d'enquêtes s'avèrent délicates. Les mêmes constatations s'imposent à la lecture des manuels d'enquêtes et questionnaires relatifs aux "relations entre partenaires". Le questionnaire destiné aux femmes (OMS, 1989 - b) ignore ainsi la possibilité d'être mariée et d'avoir, dans le même temps, un partenaire régulier ( $Q 301, p .7)$ et ne recherche aucune information sur les interruptions (volontaires ou non) de grossesse dont la connaissance revêt une grande importance pour les stratégies de prévention (la promotion du préservatif, aussi, comme moyen de contraception). De plus, une question sur les pratiques sexuelles de la femme durant les 12 derniers mois semble fort ambitieuse et risque de ne donner que des informations fragmentaires, très générales et donc inutilisables. Or, dans une perspective d'application, la nécessité de pouvoir utiliser, pour des campagnes de prévention, les résultats obtenus constitue la raison d'être de ces enquêtes quantitatives CACP et RP, qui mobilisent des moyens importants(20 000 à 100000 \$, 
entre 20 et 30 enquêteurs et 1000 à 3000 adultes interrogés : Carael, 1993, p.32 et OMS, 1989 - a) et revêtent une dimension régionale ou, le plus souvent, nationale.

Les données des enquêtes CACP : deux exemples

17 Dans le prolongement des imperfections des questionnaires administrés, les informations recueillies sur le terrain permettent d'éclairer de façon concrète les limites de la démarche et des objectifs poursuivis. Un regard sur une enquête CACP menée en avril 1990 en milieu scolaire dans la région de Banfora, au Burkina Faso (Sicard, 1992), marque les faiblesses de ce type d'étude. A dimension "régionale", ciblée sur une population donnée (adolescents scolarisés dans le secondaire), cette enquête CACP précise avoir utilisé un questionnaire "élaboré à partir des documents OMS", en particulier celui évoqué ci-dessus. La présentation des résultats par grands thèmes (connaissances; attitudes et croyances; habitudes sexuelles) ne propose aucun croisement de variables ni d'analyse des motivations. Il est ainsi regrettable de n'avoir aucune précision sur les déclarations, pour le moins paradoxales, des 124 élèves disant ne pas utiliser de préservatifs et qui, dans leur grande majorité (105 sur 124), affirment dans le même temps "avoir peur du Sida" (p.67). Sur quels choix débouche cette "peur" de la maladie, sachant qu'ils refusent le préservatif? A quels modes de transmission la peur en question est-elle rattachée ? Des questions ne sont pas posées mais, aussi, des réponses ne sont pas obtenues: "certaines investigations, comme la recherche de changement de partenaire(s) et le nombre de relations sexuelles au cours du mois précédant l'enquête sont responsables de non-réponses" (p.69). Dès lors, il apparaît difficile de soutenir que "l'élaboration du questionnaire demeure le support de toute étude (et que) son élaboration et son acceptabilité sont à la base des chances de succès de l'étude" (p.68). Si l'enquête a "très bien été acceptée par les élèves", on peut émettre l'hypothèse que l'insuffisance se situe, en amont de la confection du questionnaire, dans la définition des concepts utilisés et des attitudes décrites: par exemple, qu'entend-on par "habitude sexuelle" et "utilisation du préservatif" (à chaque rapport ? comment ?: voir les enquêtes de F. Deniaud, 1991, mentionnant les "avatars" - selon son mot - de l'emploi du préservatif).

Doris Schopper (1993) résume cette impasse méthodologique des enquêtes CACP en constatant qu'elles "essaient de quantifier des comportements qui sont "subjectifs" et dépendent d'une compréhension commune des termes qui peut être difficile à définir avec exactitude" (p.410). Parallèlement à une analyse, parfois pessimiste ("étant donné le caractère privé du comportement sexuel, il risque d'être impossible de savoir la 'vérité' au niveau de l'individu" (p.402) et souvent critique de la pertinence des enquêtes CACP, D. Schopper détaille les résultats d'une étude à laquelle elle a participé en milieu rural ougandais (entre janvier et mars 1991) et entérine des options et des résultats pourtant contestables. En complément de l'enquête strictement quantitative, des entretiens approfondis et des groupes de discussion ont ainsi été organisés. Le choix de mener les premiers avec les "responsables" de village ("village leaders") et les seconds avec les villageois - hommes d'un côté, femmes de l'autre -, n'est pas argumenté. La singularité et l'originalité des discours des "leaders" de village serait-elle plus remarquable que celle des simples habitants du village ? Ces derniers "parleraient"-ils plus facilement dans un cadre collectif que lors d'entretiens individuels ? Rien ne justifie - a priori de telles options. Par ailleurs, le constat d'un écart entre la proportion d'individus jugeant "acceptables" les relations sexuelles avant le mariage et ceux reconnaissant en avoir eu (avant leur mariage) (p.404), confirme les lacunes relevées précédemment dans le questionnaire "relations entre partenaires" 
mais n'est pas analysée. Il s'agit là d'une donnée "brute", certes stimulante mais qui peut être l'objet de diverses interprétations : discours normatif ?

Valorisation des relations sexuelles, peut-être suivant le sexe de la personne interrogée ? D'autres résultats tombent, au contraire de celui-ci, sous le sceau de l'évidence. D. Schopper nous explique ainsi, figure à l'appui, que "plus âgé est le répondant à son premier mariage, plus probables sont des relations sexuelles avant le mariage" (p.405)! Alternatives aux faiblesses des études CACP

L'étude ougandaise - à l'instar de la plupart des enquêtes CACP - met en lumière la complexité de l'interprétation des réponses brèves à des questions uniques. Le problème $\mathrm{du}$ "déclaratif' semble réellement constitutif de l'approche CACP, quantitative et portant, sans en approfondir les contradictions ou les silences, sur des thèmes relevant de l'intimité. Afin de contourner cette difficulté. D. Schopper propose de systématiser les procédures d'évaluation à partir de quelques vérifications : on se posera la question de savoir si le nombre de partenaires et le type d'union déclarés par les hommes et les femmes est concordant. Plus généralement, existe-t-il des différences significatives suivant le sexe de l'enquêteur ? Ce dernier point, au cœur de la méthodologie CACP basée sur le travail d'enquêteurs n'autorise pas de conclusions définitives. Lorsqu'elles sont interrogées par une femme, les femmes reconnaissent en plus grand nombre avoir eu des relations sexuelles les douze derniers mois que lorsqu'un homme pose la question. En revanche, elles font état d'un souhait plus important d'utiliser des préservatifs quand un enquêteur homme le leur demande $(33,1$ $\%$ contre $13,7 \%$ en réponse à des enquêtrices). Le contenu de l'étude ne permettant pas - faute de "croisements" de réponses" - de juger de la validité des réponses fournies, la proposition d'évaluer en général ces enquêtes CACP (Carael, 1993, p.34) et, plus précisément, ces réponses divergentes, au moyen de recherches spécifiques (Schopper, 1993, p.411), peut sembler opportune. Ce choix évite cependant de s'interroger sur le contenu même de la question ayant biaisé les réponses.

À cet égard, une étude zambienne en milieux scolaire et universitaire (1121 personnes interrogées, début 1990; Mufune, 1993), reprenant quelques éléments des enquêtes CACP (perceptions de la sexualité et du Sida) apporte un élément de solution en ne posant pas certaines questions. Les élèves et étudiants ne sont donc pas interrogés sur leur vie sexuelle -"questions trop sensibles et qui n'amèneraient pas de réponses sincères" (pp.25-26) - mais sur celle de leur "meilleur ami". On pourrait craindre qu'un tel détour donne l'occasion de sur-évaluer la sexualité des personnes en question (le "meilleur ami") : les conclusions s'opposant aux affirmations de J. Caldwell (1989) - par ailleurs fortement critiquées - sur un encouragement de la "permissivité et la promiscuité sexuelle en Afrique" révèlent au contraire une perception négative du multi-partenariat sexuel et des relations sexuelles hors-mariage. Il convient de se garder de généralisations à partir d'études ciblées "socialement" (élèves-étudiants) : ce seul témoignage suffit, toutefois, à relever des jugements erronés sur une prétendue sexualité africaine même si, en retour, il ne permet pas de brosser un tableau complet des comportements sexuels, ne serait-ce qu'au seul niveau de la Zambie. La richesse et l'ambiguïté des démarches qualitatives et, en particulier, anthropologiques se situe, me semble-t-il, dans une capacité à indiquer des erreurs d'interprétation à ne pas commettre et des précautions conceptuelles à prendre allant de pair avec une difficulté - revendiquée et assumée -proposer des solutions claires et des conduites à tenir.

Des concepts aux méthodes : la négligence d'un lien 

difficile insertion du regard anthropologique dans les études de l'OMS, un problème central vient compliquer le rapprochement entre les disciplines et acteurs de la recherche et de l'intervention sur le Sida autour des protocoles de l'OMS. Nous souhaitons revenir ici sur la question des concepts utilisés lorsque des groupes et des comportements sont étudiés. La notion de "groupes à risque", par son caractère stigmatisant pour ces derniers et inexact sur le plan épidémiologique, a été délaissée au profit de celle de "comportements à risque". Les Sciences sociales - dites "comportementales" à l'OMS - en contribuant à ce déplacement de l'objet de recherche au plus près des pratiques des individus, indépendamment d'un quelconque "statut" (une prostituée utilisant systématiquement des préservatifs n'est pas "à risque" de contracter le VIH), ont été insuffisamment sollicitées pour accompagner cette évolution d'une réflexion sur la nature des concepts employés.

en est ainsi de la prostitution. Comme le fait justement remarquer H. Standing, en uniformisant des pratiques et des situations variées sous l'expression de "sexe commercial" ou "rémunéré", l'OMS - sur le plan de la conceptualisation du phénomène - s'interdit de réfléchir aux critères économiques présidant à sa définition, à la conscience des intéressées d'être ou non "prostituées" et aux perceptions des hommes de ces pratiques (1992, p.477). Compte-tenu des écueils rencontrés par les recherches dans le milieu de la prostitution, une telle réflexion initiale devrait s'imposer.

Fort éloignée, de par la méthodologie déployée, des études quantitatives soutenues par l'OMS, la recherche approfondie menée par H. Pickering (1992) auprès des prostituées et de leurs clients en Gambie durant 14 mois expose avec franchise le délicat travail de terrain qui la sous-tend et les limites des investigations, y compris anthropologiques, sur les comportements sexuels. Avant d'en venir au contenu même de la démarche adoptée, il importe de préciser, qu'à la différence de nombreux textes sur les aspects sociaux du Sida, le travail de H. Pickering s'attarde longuement sur l'exercice du terrain, les conditions de réalisation de l'enquête. Loin d'être un simple exercice de style obligé, standardisé, formalisé en quelques phrases, la présentation des modalités de la recherche éclaire fondamentalement la problématique de l'auteur. Les sciences sociales ne peuvent faire l'économie de ce travail critique, en particulier sur des thèmes où la qualité de la relation établie avec les interlocuteurs est un gage d'exhaustivité et de cohérence des informations recueillies, à défaut de leur "véracité", difficilement attestable avec certitude. Ainsi, malgré des rencontres individuelles avec les prostituées - dont "la majorité devinrent rapidement des participantes enthousiastes" (p.76) - et la multiplication des approches (questionnaire, bilan des relations sexuelles quotidiennes, observation et entretiens approfondis), celles-ci donnèrent, dans un premier temps, de faux noms, occultèrent des avortements et l'utilisation de drogues et inventèrent des histoires familiales. Seule la comparaison des réponses et la répétition des entretiens - en dehors du seul canevas d'un questionnaire "fermé" - permettent d'élucider ces points. Subsistent toutefois des imprécisions sur les activités sexuelles des prostituées (le prix du rapport sexuel) et leur fréquence (nombre de clients) (p. ? ?). Par ailleurs, le travail auprès de clients, effectué au moyen d'un bref questionnaire du fait de leur manque de disponibilité, ne peut faire l'objet d'une évaluation rigoureuse $\mathrm{H}$. Pickering reconnait les limites de cette partie de la recherche, précisant cependant qu'elle faillit ne pas avoir lieu, les prostituées craignant initialement que les informations données par leurs clients sur les prix payés ne restent pas confidentielles.

Bulletin de l'APAD, 8 | 1994 

"qualitatif et le "quantitatif" ne suffit pas à expliquer la pertinence des résultats obtenus. L'option méthodologique ne peut se définir qu'en fonction des objectifs recherchés et, de ce fait, par une réflexion préalable sur la nature des concepts et pratiques au cœur de la problématique. Pas plus que l'on ne reprochera à l'épidémiologie sa démarche comptable, essentiellement quantitative, on ne fera grief à l'anthropologie de prôner une approche longue, empirique et comparative de phénomènes et de groupes circonscrits dans l'espace, à condition que l'objet d'étude l'exige. Ce qui peut sembler ici une évidence, reste très souvent négligé, comme nous l'ont montré les précédents exemples d'enquêtes CACP qui n'adaptent pas - sur certains points - leurs objectifs à leurs méthodes ${ }^{2}$. En se penchant sur les questions de "validation" des enquêtes quantitatives, M. Boulton (1993) confirme les dangers de ce hiatus entre méthodes et objectifs. Pour pouvoir prétendre analyser les changements de comportements, les études de grande envergure sur la sexualité doivent inclure des procédures d'évaluation (p.251). Or, le plus souvent, la méthodologie des approches quantitatives, omettant des investigations ultérieures visant à évaluer les informations recueillies, ne se donnent pas les moyens d'appréhender les processus de transformation, au centre de toute analyse anthropologique. M. Carael (1993) regrette, à cet égard, l'absence de répétition des enquêtes CACP à quelques années d'intervalle, afin d'approcher les changements de comportements (p.33). Scientifiquement souhaitable, la reprise d'une étude quantitative, financièrement et techniquement lourde à gérer, correspond-elle à une véritable volonté politique, au niveau de l'OMS et des PNLS (Programmes nationaux de lutte contre le Sida)? En effet, dans leur conception même - offrir un éclairage, ciblé dans le temps et représentatif à l'échelle du pays, sur un ensemble d'attitudes et de représentations liées au Sida - les enquêtes CACP n'incluent pas la nécessité d'une évaluation. Les PNLS d'autre part, les sollicitent afin de lancer des campagnes de prévention: les répéter, ne serait-ce pas admettre implicitement leur faillite?

La solution à ce problème de l'évaluation se situe en partie dans la succession d'une étude qualitative à l'enquête CACP. Une initiative de cet ordre a été expérimentée en Ouganda, dans le district de Rakaï, où, en complément d'une enquête CACP classique, des "focus group" (groupes de discussion) ont été organisés (Kondé-Lule, 1993). Dans une perspective anthropologique, l'intérêt de cette progression de la réflexion réside moins dans l'utilisation en tant que telle de "focus group" que dans la possibilité de "générer de nouveaux domaines d'investigation et des questions qui peuvent être inclus dans des questionnaires et activités ultérieures" (p.679). Parmi ces "activités", nous pourrions ranger des recherches anthropologiques que les premiers résultats des "focus group" sur les perceptions du Sida, du préservatif ou du dépistage rendent pertinentes. C'est à un projet de ce type que se sont attelés des anthropologues américains et ougandais s'intéressant aux réactions et réponses familiales au Sida (McGrath, 1993). Le suivi de 22 familles - dont un des membres est séropositif - de l'agglomération de Kampala, pendant 5 mois (de mars à juillet 1989), n'exclue pas des comparaisons avec des situations observées dans d'autres contextes. Ainsi, à l'image de nos observations à Abidjan (Vidal 1992-a el b; 1993), les malades de Kampala rencontrés informent sélectivement leurs proches, perçoivent des risques d'exclusion de l'entourage -même s'ils ne vivent pas concrètement des rejets - fondés sur une "suspicion" de séropositivité. 
27 Nous pourrions multiplier les similitudes d'analyse de l'épreuve de la maladie, entre Abidjan et Kampala: contentons- nous de relever que la démarche anthropologique appliquée dans toute sa spécificité autorise des comparaisons -loin d'être réservées aux seules études quantitatives - et, plus encore, les suggèrent avec force, comme cet exemple le montre clairement. Par spécificité de l'approche anthropologique nous entendons, aussi bien, le recul dans le temps par rapport à l'objet de recherche permettant d'observer des transformations d'attitudes, que la prise en considération des discours individuels dans leur totalité, n'excluant aucun des thèmes qui définissent les relations sociales, à l'intérieur de la famille et des réseaux amical et professionnel.

Sur ce point, le travail de J.W. MacGrath et de ses collègues, malgré sa relative brièveté (cinq mois) ne s'apparente pas une étude de type RAP. Les "rapid assessment" (ou "anthropological") procedures" se caractérisent en effet par leur focalisation sur ce qu'A. Desclaux appelle des "données objectives" (comportements, conditions de vie...), laissant à l'anthropologie classique les données "conceptuelles", "délicates" (représentation, peurs...) (1992, p.302). Ceci étant, le choix d'entretiens approfondis de préférence aux questionnaires fermés ou semi-ouverts les distingue nettement des enquêtes CACP. Originale à la fois au regard des approches RAP et CACP, la recherche actuelle en anthropologie sur le Sida en Afrique fournit donc divers exemples d'études qui - sur des terrains éloignés les uns des autres - donnent corps à un ensemble de connaissances comparables (mentionnons, en plus des textes déjà cités, ceux de M. Ankrah en Ouganda - 1992 - et de C. Raynaut et F. Muhongayire au Rwanda, 1993) et parfois même généralisables, contrairement aux idées reçues sur les approches "qualitatives" .

L'anthropologie face aux enjeux éthiques et méthodologiquesÉthique du chercheur, éthique de la recherche

En filigrane des réflexions précédentes sur les méthodes de recherche anthropologiques et celles émanant plus particulièrement des institutions de financement de lutte contre le Sida, la dimension éthique des interventions et travaux de terrain revient constamment dans les propos des chercheurs, à la fois comme un passage obligé des études et un point d'achoppement dans les rapprochements interdisciplinaires. Avant de présenter les principaux éléments de ce débat, la mise en garde quelque peu provocatrice de B. Hoffmaster (1992) - qui ne concerne pas spécifiquement le Sida mais peut lui être appliquée - selon laquelle "quand tout devient éthique, le danger est que l'éthique ne devienne rien" (p.1424) me paraît d'actualité. En effet, l'inévitable réflexion éthique présidant à toute intervention (non seulement au niveau de la recherche mais aussi dans la communication et l'information) dans le domaine du Sida doit se concentrer sur la protection de l'individu au cœur de la recherche, qu'il soit malade, proche de patient séropositif ou personne susceptible d'avoir un "comportement à risque". Il ne s'agit certes là que d'un aspect du "discours éthique" habituel fondé, comme nous le rappelle G. Seidel (1993, p.182), d'une part, sur ce qui est "bien pour le plus grand nombre", et d'autre part, sur le respect de la dignité et des droits de l'homme. En somme, cela revient à promouvoir des mesures destinées à protéger conjointement la société (le "plus grand nombre") et l'individu. Si les premières peuvent aller dans le sens d'une plus grande équité - par l'information et l'éducation dont on perçoit chaque jour le caractère déterminant dans les actions contre le Sida - elles comprennent aussi des mesures de protection, coercitives, à l'image des quarantaines sanitaires. 
30 Au niveau de l'individu, le principal enjeu éthique demeure le respect de la confidentialité, droit fondamental et à mon sens premier de la personne, dans toute recherche sur le Sida. Sans certitude de confidentialité, aucune information ne peut être donnée par le praticien ni assimilée par le patient. Exiger le respect de la confidentialité sous-entend - ipso facto - la recherche du consentement éclairé des sujets de l'étude : la première ne peut être obtenue au détriment du second. D.G. Gréco (1992) considère à juste titre que les personnes "incompétentes pour donner légalement leur consentement informé (...) peuvent être considérées comme vulnérables" (p.128). Ajoutons qu'il nous semble que cette "vulnérabilité" concerne tout individu lorsque le consentement puis les assurances de confidentialité sont bâclés ou non respectées pour ces dernières, et pas uniquement "les enfants, les femmes enceintes et allaitantes, les malades mentaux, les personnes psychiquement instables et les prisonniers" (p.128).

31 Ces considérations renvoient certes, en premier lieu, à une éthique de la pratique médicale qui déborde du cadre déontologique habituel du fait de ses implications pour la prise en charge familiale du patient. Ceci précisé, l'inévitable collaboration d'un grand nombre de recherches en sciences sociales avec le milieu médical - ne serait-ce que par l'utilisation des services des structures sanitaires - transforme rapidement ces questions d'éthique médicale face au Sida en enjeux éthiques pour la recherche, en général. Évoquant la mise en œuvre de mon travail auprès de séropositifs à Abidjan (Vidal, 1992-c), je me suis penché sur le difficile accord entre perspectives anthropologique, épidémiologique et médicale. L'éthique de la prise en charge du séropositif est ici au cœur du fonctionnement de la structure de soins- (les Centres anti-tuberculeux). En effet, dans le contexte de 1990 - qui a depuis lors évolué l'absence de "prétest" et la non-annonce de la séropositivité (sauf rares exceptions) et mon souhait de rencontrer et d'établir des relations durables avec des patients éveillait chez les épidémiologistes en charge de "cohortes" de séropositifs la crainte de voir certains de "mes" malades abandonner "leur" suivi. Par ailleurs, je me suis retrouvé dans la position ambiguë de côtoyer des patients qui ignoraient leur statut sérologique. Ne désirant pas contourner cette difficulté en ne travaillant qu'avec les - rares patients informés de leur séropositivité, la question s'est posée de l'attitude à adopter face à la sous-information sur la nature de la maladie et, le cas échéant, aux demandes d'explications de certains patients. A de rares exceptions près pour lesquelles la demande du séropositif était pressante et s'accompagnait d'indices précis d'abandon des mesures de prévention, il a été décidé de ne pas assumer la révélation de la séropositivité, demeurant de la responsabilité du médecin (éventuellement des services sociaux de la structure sanitaire) ayant décidé le dépistage.

Nous voyons donc clairement qu'un choix éthique personnel, portant sur un petit nombre de patients, est aussi l'expression et le fruit d'une situation d'ensemble (les procédures de prise en charge en vigueur dans les CAT de 1990 à aujourd'hui). Il est illusoire de vouloir distinguer, dans le domaine éthique, les dimensions personnelles et organisationnelles, celles de la recherche et de l'exercice de la médecine (y compris le travail des assistantes sociales). Sauf à isoler l'anthropologie du Sida des acteurs de la médecine et des structures de soins, mie éthique globale est à imposer qui nécessite des remises en question à la fois pour le médecin (renouer avec un véritable dialogue avec le patient, obtenir son consentement pour le test, l'informer des résultats quels qu'ils 
soient) et l'anthropologue (la position neutre et objective par rapport au Sujet ne devient-elle pas inadaptée ?).

De plus, l'expérience d'Abidjan nous le montre la concertation sur une éthique commune ne pourra faire l'économie d'un débat méthodologique. De la prise en charge du plus grand nombre possible de patients - ce qui équivaut avec un personnel constant - à consacrer moins de temps à l'information du malade et à l'annonce du diagnostic au respect des "cohortes" des épidémiologistes et de l'approche anthropologique, longue et interpersonnelle, les "traditions" et nécessités méthodologiques propres à chaque discipline ne facilitent pas un consensus éthique. Pour tenter de l'élaborer, une réflexion préalable de chaque discipline sur les effets du Sida sur sa propre démarche s'avère indispensable.

Problèmes et implications épistémologiques

Partie intégrante du métier d'anthropologue, la collaboration avec un enquêteur-interprète peut revêtir des inconvénients au regard des enjeux de confidentialité évoqués précédemment. M. Boulton (1993) estime que l'établissement de relations durables entre l'enquêteur local (elle parle, en général, d"'indigenous worlker") et son interlocuteur est susceptible d'amener des révélations d'informations en principe confidentielles (p.250). La proximité culturelle (appartenance à la même "ethnie") constituerait un handicap au regard des exigences méthodologiques et éthiques de la démarche anthropologique. S'il n'est pas original en anthropologie de par le rôle de médiation de l'interprète, ce problème revêt une acuité particulière dans le cas du Sida, maladie sur laquelle pèsent toujours honte et soupçon de "faute". Le danger réside, me semble-t-il, dans le constat qu'à la position intermédiaire (entre l'anthropologue et son sujet) de l'enquêteur-interprète, s'ajoute celle de l'anthropologue lui-même, entre médecin et malade. En effet, à l'écoute des représentations de la souffrance de l'un et des interrogations de l'autre sur sa pratique professionnelle, l'anthropologue travaille au cœur de sollicitations qui constituent, paradoxalement, un de ses centres d'intérêt. Ainsi, la connaissance de la maladie, recueillie et analysée telle que le patient la donne à voir et à comprendre, se trouverait bien évidemment bouleversée par une révélation émanant de l'anthropologue. $\mathrm{n}$ en serait de même du rapport du patient à l'institution médicale, devenue un objet de méfiance car ayant caché la "vérité" sur la maladie. La difficulté, déjà notée, d'une telle situation se retrouve dans le débat récurrent en anthropologie sur l'implication du chercheur qui doit éviter de créer des situations à observer au risque - assumé par certains - de voir cet objet de recherche changer de nature.

Parmi les questions épistémologiques posées par le Sida se trouve, en amont et en complément du rôle de l'enquêteur évoqué ici, celle de la relation avec l'objet de recherche. Aussi précise soit leur identification (telle catégorie de prostituées, de jeunes, de malades), les individus ou groupes rencontrés obligent le chercheur à une réflexion sur son travail. Là encore, il ne s'agit pas d'une difficulté spécifique à la thématique du Sida : elle prend toutefois une dimension particulière, originale, dans la mesure où une expérience relevant de l'intimité (la souffrance des séropositifs; la sexualité en général) est au centre de l'observation de l'anthropologue et de l'échange qu'il génère. Un déplacement s'opère de la classique non-divulgation d'informations liée à la préservation d'un secret, correspondant lui-même fréquemment à un espace sacré, vers la préservation de préoccupations profondément intimes de l'individu. L'investigation anthropologique atteint là les limites fixées par sa méthodologie et sa 
conception de la relation à l'autre. Travailler auprès de malades du Sida dépasse les acquis de l'expérience de terrain sur des savoirs protégés (comme ceux des guérisseurs, par exemple) du fait de la permanence du thème de la mort et, plus encore, de la douleur, aussi bien physique que psychologique du séropositif.

Dans ce débat sur l'intervention du chercheur (introduit autour de la question du partage du diagnostic) l'anthropologue se trouve, d'autre part, rétrospectivement confronté à des démarches promouvant l'analyse des aspects surnaturels du traitement de la maladie. B.G. Schoepf (1991) et R Packard - P. Epstein (1991) dénoncent à cet égard certains "mauvais usages" de l'anthropologie (Schoepf, p.757) où l'exotisme de micro-observations datées conforte les préjugés - repris les premières années de l'expansion de l'épidémie en Afrique - sur une "promiscuité sexuelle culturellement déterminée" (Packard, p.775).

Le fait que certains anthropologues aient utilisé des analyses de ce type pour répondre aux interrogations initiales de la recherche epidémiologique et biomédicale sur les facteurs de risque de diffusion du Sida, traduit l'ambiguïté et la relation de l'anthropologie à l'objet de recherche-Sida. Celle-ci ne peut être levée que si la discipline se penche sur ses anciens terrains de recherche et en actualise les données recueillies, à la lumière de la problématique du Sida. La crédibilité du savoir anthropologique constitué autour des fondements et des implications du Sida est ici en jeu. Le Sida impose un recul par rapport aux vieilles monographies ethnologiques dont les sciences biomédicales, par facilité mais aussi faute de données comparables, actuelles, s'approprient les résultats au nom de la recherche anthropologique. Un tel mouvement témoigne d'un affaiblissement de la "capacité interprétative", notée par G. Balandier, qui va de pair avec un renforcement des moyens d'investigation de la recherche (1993, p.302). G. Balandier conclut alors que "c'est à cette crise de l'interprétation qu'il faut vouloir remédier" et ajoute "alors, le désir d'appartenir à ce monde-ci deviendra plus vif - et les actions qu'il nourrit seront moins aveugles" (p.302). Cette ultime remarque nous rappelle avec à propos que la faiblesse analytique et la relative inefficacité pratique, dans le domaine de la prévention, des études standardisées - type CACP - ainsi que le manque d'innovation épistémologique de l'anthropologie sur le thème du Sida ont créé un "aveuglement" que seule une réflexion conjointe sur les méthodes employées et les objectifs recherchés permettra d'atténuer.

\section{BIBLIOGRAPHIE}

Althabé G., 1993, "Vers une ethnologie du présent" pp.89-98, in Les nouveaux enjeux de l'anthropologie. Autour de Georges Balandier (ss. dir. de G. Gosselin), L'Harmattan, 302 p.

Ankrah M.E. (1991). "AIDS and the social side of health", Social Science et Medicine, 32, 9, 967-980.

Augé M., 1984, "Ordre biologique, ordre social. La maladie forme élémentaire de l'événement" in : Augé M., Herzlich C. (Ed.), Le sens du mal, Editions des Archives Contemporaines, 35-91.

Augé M., 1986, "L'anthropologie de la maladie", L'Homme, 11, 2, 81-89. 
Balandier G., 1993, "Où il est question de modernité", pp. 295-302, in : Les nouveaux enjeux de l'anthropologie. Autour de Georges Balandier (ss. dir. de G. Gosselin), L'Harmattan, 302 p.

Boulton M., 1993, "Methodological issues in HIV,'AIDS social research : recent debates, recent debates, recent developments", AIDS, 7 (Suppl. 1), S249-S255.

Caldwell J., Caldwell P, Quiggin P., 1989, "The social context of AIDS in Sub Saharan Africa", Population and Development Review, 15, 185.

Carael M., 1993, "Bilan des enquêtes CAP menées en Afrique : forces et faiblesses" pp.27-36, in : Les Sciences sociales face au Sida : cas africains autour de l'exemple ivoirien, Actes de l'Atelier "Les Sciences sociales face au Sida" (Dozon J.P. et Vidal L, Ed. Sc.), Bingerville (Côte d'Ivoire), 15-17 Mars 1993, Centre ORSTOM de Petit-Bassam, 391p.

Deniaud F., 1991, "Sida, préservatifs et jeunesse urbaine en Côte d'Ivoire. Un essai d'ethnoprévention" Bulletin de liaison CNDT. Hors-série, 46-69.

Desclaux A., 1992, "Les méthodes rapides en anthropologie", Cahiers Santé, 5, 2,30-300.

Dozon J.P., 1983, "L'anthropologie médicale : fabrication d'un nouveau regard", Sciences Sociales et Santé, 3-4, Septembre, 37-41.

Dozon J.P., Fassin D., 1989, "Raison épidémiologique et raisons d'Etat. Les enjeux sociopolitiques du sida en Afrique", Sciences Sociales et Santé, 7, 1. 71-36.

Greco D., 1992, "Ethique, pauvreté et Sida", Cahiers Santé, 2, 2 122-129.

Green E., 1992, "Sexually transmitted disease, ethnomedicine and health policy in Africa', Social Science and Medicine. 35. 2 121-130.

Hoffmaster B., 1992, 'Can ethnography save the life of medical ethics?, Social Science and Medicine, 35. , 1421-31.

Hours X. (ss dir de), 1993, "Aspect des systèmes de santé dans le sud", Sciences Sociales et santé, 11. 2, Juin. 146- p.

Jamard J.L., 1989-90, "Parménide, Héraclite et l'anthropologie française", 2ème partie, Gradhiva". 39-72.

Kalaora B., 1989, "Paul Descamps ou la sociologie leplaysienne à l'épreuve du Portugal de Salazar", Gradhiva, 6, 50-64.

Konde-Lule J., Musagara M., Musgrave S., 1993, "Focus group interviews about AIDS in Rakai district of Uganda", Social Science and Medicine, 37, 5, 679-84.

Mann J., 1993, "Santé et Droits de l'Homme : défi de la Santé Publique moderne", Congrès d'épidémiologie et de Santé Publique, Bordeaux 27-30 septembre, Revue de presse Sida, Université de Bordeaux II, 4, 7-8, 493- 702.

McGrath J., Ankrah E.M., Schumann D., Nkumbi S., Lubega M., 1993, "AIDS and the urban family. its impact in Kampala, Uganda" AIDS Care, 5, 1,55-70.

Mufune P., Osei-Hwedie K., Mwensa L., 1993, "Attitudes toward risky sexual behaviour and reactions to people infected with HIV among zambian students", International Family Planning Perspectives 19, 1 25-7.

OMS, 1989 - a, Research package : Partner relation and risk of HIV infection : Phase 1, GP A SBR, November. 
OMS, 1989 - b, Partner relation and risk of HIV infection : Phase 1, OPA-SBR, November, Questionnaire Femmes.

OMS, 1989 - c, Partner relation and risk of HIV infection : Phase 1, OPA/SBR, November, Questionnaire Hommes.

OMS, 1989 - d, Research package : Knowledge, attitudes, beliefs and practices on AIDS, Phase 1, Appendix : questionnaire, November, OPA/SBR, $28 \mathrm{p}$.

OMS, 1990 - a, Research package : knowledge, attitudes, beliefs and practices on AIDS, Phase 1, November, OPA/SBR, 26.1.90.

Packard R., Epstein P., 1991, "Epidemiologists, social scientist and the structure of medical research on AIDS in Africa", Social Science and Medicine 33. 7, 771-794.

Pickering H., Todd J., Dunn D., Pepin J., Wilkins A., 1992, "Prostitutes and their clients : a gambian survey", Social Science and Medicine, 34, 1, 75-88.

Pina Cabra J. De, 1992, "Anthropologie et identité nationale au Portugal", Gradhiva, II, 31-46.

Piol P., T. Quinn, H. Taelmam, F. Feinsod, N. Mbendi, P. Mazebo, K. Ndangi, W. Stevens, Kalambayi, S. Mitchell, C. Bridts J. McCormick, 1984, "Acquired immunodeficiency syndrome in a heterosexual population in Zaire", Lancet, n. 65-9.

Puccini S., 1991, "Institutionnalisation de l'anthropologie italienne au XIXe siècle", Gradhiva, 9, 63-76.

Raynaut C., Muhongayire F., 1993, "Chronique d'une mort annoncée : problèmes d'éthique et de méthode posés par l'application de la démarche anthropologique au suivi de familles touchées par le sida (à partir du cas rwandais)", pp.299-322, in : Les Sciences sociales face au Sida : cas africains autour de l'exemple ivoirien, Actes de l'Atelier "Les Sciences sociales face au Sida" (Dozon J.P. et Vidal L, Ed. Sc.), Bingerville (Côte d'Ivoire), 15-17 Mars 1993, Centre ORSTOM de Petit-Bassam, 391p.

Schoepf B., 1991, "Ethical, methodological and political issues of AIDS research in Central Mrica", Social Science and Medicine, 33, 7, 749-763.

Schopper D., Doussantousse S., Orav J., 1993, "Sexual behaviors relevant to HIV transmission in a rural african population. How much can a KAP survey tell us ?", Social Science and Medicine, 37, 3, 401-12.

Seidel G., 1993, "The competing discourses of HIV/AIDS in Sub-saharan Africa : discourses of rights and empowerment vs dicourses of conuol an exclusion", Social Science and Medecine, 36, 3, 175-194.

Sicard J.M., Kanon S., Ouedraogo L.A., Chiron J.P., 1992, "Evaluation du comportement sexuel et des connaissances sur le Sida en milieu scolaire au Burkina Faso", Annales de la Société Belge de Médecine tropicale, 72, 63-72.

Sindzingre N., Zemp1eni A., 1982, "Anthropologie de la maladie", in : Godelier M., Les Sciences de l'Homme et de la Société en France, La Documentation Francaise.

Standing H., 1992, "AIDS : conceptual and methodological issues in researching sexual behaviour in sub-saharan African, Social Science and Medicine, 5, 475-483.

Vidal L., 1992-a, "Sida et représentations de la maladie ; éléments de réflexion sur la séropositivité et sa prise en charge (Abidjan, Côte d'Ivoire)", Cahiers des Sciences Humaines, 28, 1, 83-98. 
Vidal L., 1992-b, "Itinéraire thérapeutique et connaissance de la maladie chez des patients séropositifs pour le VIH (Abidjan, Côte d'Ivoire)", Cahiers Santé, 2, 5, Septembre - Octobre, 312-3 21.

Vidal L., 1992-c, "L'épreuve du Sida : une anthropologie entre éthique et terrain", Journal des Anthropologues, $\mathrm{n}^{\circ}$ 50-51, pp.121-128.

Vidal L., 1993, "Le Sida et l'anthropologue : une rencontre à risques (à propos d'une recherche anthropologique sur les représentations et la prise en charge du Sida à Abidjan)", Chroniques du SUD ORSTOM, Mai, 128-134.

Zempleni A., 1982, "Anciens et nouveaux usages sociaux de la maladie en Afrique", Archives de Sciences Sociales des Religions, 14-1, Juillet- Septembre, 5-19.

\section{NOTES}

1.Au détriment de données très partielles sur des comportements sexuels qui ont contribué à façonner la thématique scientifiquement inexacte et réductrice, et politiquement dangereuse d'une prétendue "sexualité africaine" .

2.H. Standing ( 1992 - développe largement l'importance de ce lien direct entre conceptualisation et choix méthodologique. 